\title{
Performance Evaluation of the MMSE Delayed Decision Feedback Sequence Detector
}

\author{
Maurizio Magarini, Arnaldo Spalvieri, and Guido Tartara, \\ Dipartimento di Elettronica e Informazione, Politecnico di Milano, \\ Piazza Leonardo da Vinci, 32, I-20133 Milano (Italy).
}

\begin{abstract}
In signal equalization, a technique that allows reduction of the number of states of the Viterbi detector is the Delayed Decision Feedback Sequence Detector (DDFSD). In order to achieve good performance, it is crucial to operate an appropriate prefiltering of the received sequence before the DDFSD. This paper is devoted to performance evaluation of the DDFSD when the prefilter of a minimum mean square error decision feedback equalizer is adopted. The union upper bound is used to evaluate the probability of first-event error, and truncation of the sum to the error sequences that dominate the performance is discussed.
\end{abstract}

\section{INTRODUCTION}

The Delayed Decision Feedback Sequence Detector (DDFSD) is an equalization scheme based on the sampled matched filter, a prefilter, and a Viterbi algorithm where the channel memory is truncated. The performance loss due to memory truncation is mitigated by a per-survivor processing [1], where the past history of each survivor is used in a DFE scheme. In the DDFSD originally proposed in [2], the noise whitening prefilter was adopted (W-DDFSD). In [3] it is proposed to adopt the feedforward prefilter of a Minimum Mean Square Error (MMSE) Decision Feedback Equalizer (DFE), while in [4] the cascade of the matched filter and of the MMSE prefilter is realized as a fractionally spaced finite impulse response filter. In [5] it was shown that, without complexity reduction in the Viterbi algorithm, the MMSE prefilter leads to Maximum Likelihood Sequence Detection (MLSD) with minimum number of states, and that, when the DDFSD is considered, the MMSE-DDFSD allows to improve over the WDDFSD. In this paper, performance evaluation of the MMSEDDFSD is addressed.

\section{SYSTEM MODEL}

We consider the model of a binary uncoded data sequence transmitted over a baseband linear channel corrupted by zeromean additive white Gaussian noise. The receiver consists of the sampled matched filter, a prefilter, and a sequence detector. The block diagram of the system is reported in Fig. 1. With reference to the figure, we assume in the following that $\tilde{a}_{k} \in\{-1,+1\}$, and that the two-sided power spectral density of the AWGN is $\sigma^{2}$. The impulse response of the system from the source to the output of the sampled matched filter is represented by the $z$-transform $r(z)=\sum_{k=-\nu}^{\nu} r_{k} z^{-k}$, where $z^{-1}$ represents the unit delay.

Among sequence detectors, we are interested in the DDFSD

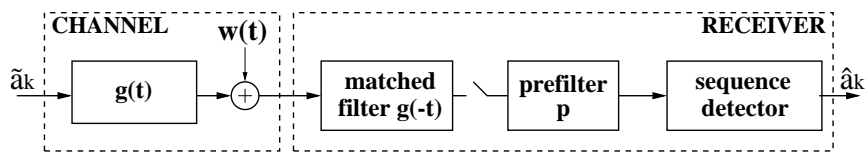

Fig. 1. Channel and receiver block diagram.

of [2]. The DDFSD is a Viterbi algorithm with $2^{\mu}$ states, $0 \leq$ $\mu \leq \nu$, where the metric of each survivor is calculated using a DFE with $\nu-\mu$ taps. In the DDFSD originally proposed in [2], the noise whitening prefilter was adopted. In consideration of the success that the MMSE-DFE had since the classical paper of Salz [6], we feel that it calls for the MMSE-DDFSD. The MMSE-DDFSD is based on the spectral factorization

$$
d(z) d\left(z^{-1}\right)=r(z)+\sigma^{2}
$$

where that $d(z)$ that is causal and minimum phase is taken. The prefilter is

$$
p(z)=\frac{d(z)}{r(z)+\sigma^{2}}=d^{-1}\left(z^{-1}\right) .
$$

Note that $\sigma>0$ guarantees the existence of both $d(z)$ and $p(z)$ [7]. The metric of the transition that diverges at time $k-1$ from state $\left(a_{k-\mu}, \ldots, a_{k-1}\right)$ and merges at time $k$ in state $\left(a_{k-\mu+1}, \ldots, a_{k}\right)$ is

$$
\begin{aligned}
& b_{k}\left(a_{k-\mu}, \ldots, a_{k}\right)=\left(x_{k}-\sum_{j=0}^{\mu} d_{j} a_{k-j}\right. \\
& \left.-\sum_{j=\mu+1}^{\nu} d_{j} \hat{a}_{k-j}\left(a_{k-\mu}, \ldots, a_{k-1}\right)\right)^{2}-\sigma^{2} a_{k}^{2},
\end{aligned}
$$

where $x_{k}$ is the $k$-th sample at the output of the prefilter, and $\hat{a}_{k-j}\left(a_{k-\mu}, \ldots, a_{k-1}\right)$ is the estimate of the $(k-j)$-th bit present in the survivor that at time $k-1$ merges in the state $\left(a_{k-\mu}, \ldots, a_{k-1}\right)$. Note that, for binary transmission, the term $-\sigma^{2} a_{k}^{2}$ is common to all the metrics and can be omitted. It is shown in [7] that (1) and (2) minimize the MSE,

$$
\mathrm{MSE}=E\left\{u_{k}^{2}\right\}
$$

In (4), $E\{\cdot\}$ denotes the expected value, and

$$
u_{k}=x_{k}-\sum_{j=0}^{\nu} d_{j} \tilde{a}_{k-j},
$$


is the $k$-th sample of the distortion sequence. We emphasize that the two extreme cases of the MMSE-DDFSD are MLSD with minimum number of states for $\mu=\nu$ [5], and the MMSEDFE of [7] for $\mu=0$. The W-DDFSD of [2] is obtained putting $\sigma^{2}=0$ in (1), (2), and (3).

\section{PERFormance EVAluation}

Neglecting error propagation, Sheen and Stüber have derived in [8] a close approximation to the Bit Error Rate (BER) of the W-DDFSD. To our best knowledge, no upper bound on the error performance of the DDFSD is published in the open literature. In what follows, it is shown that the First-Event Error Rate (FEER) of the DDFSD, can be upperbounded by the union bound. Roughly speaking, the FEER measures the probability of error burst. More precisely, the FEER is a conditional probability. Without loosing generality, we assume that the event is $e_{0} \neq 0$, where

$$
e_{k}=\hat{a}_{k}-\tilde{a}_{k},
$$

$\hat{a}_{k}$ being the $k$-th decision of the sequence detector, and that the condition is

$$
[e(z)]_{-\nu}^{-1}=0
$$

where the notation $[x(z)]_{i}^{j}=\sum_{k=i}^{j} x_{k} z^{-k}$ is used. Note that condition (6) is different from the condition imposed in [8], where $[e(z)]_{-\mu}^{-1}=0$ is considered. The union upper bound on the FEER is [9]:

$$
\text { FEER } \leq \sum_{e(z) \in \mathcal{E}} 2^{-w_{e}} P(e)
$$

In (7), $w_{e}$ is the Hamming weight of $e(z)$,

$$
w_{e}=\frac{\left[e(z) e\left(z^{-1}\right)\right]_{0}}{4},
$$

and $\mathcal{E}$ is the set of error polynomials having the form

$$
e(z)=\sum_{k=0}^{l-1} e_{k} z^{-k}, \quad l=1,2, \cdots
$$

where $e_{0} \neq 0, e_{l-1} \neq 0$, and there are no more than $\mu-1$ consecutive zeros between 0 and $l-1$. The probability $P(e)$ appearing in (7), which is hereafter called pairwise error probability, is the error probability in the binary test between $[\tilde{a}(z)]_{0}^{l-1}$ and $[\tilde{a}(z)+e(z)]_{0}^{l-1}$. Note that the binary test may take place only if the two events (6) and

$$
[e(z)]_{l}^{l+\mu-1}=0
$$

occur. Actually, in the DDFSD, the binary test between $[\tilde{a}(z)]_{0}^{l-1}$ and $[\tilde{a}(z)+e(z)]_{0}^{l-1}$ takes place when the two competitors merge for the first time in the reduced trellis, that is when (9) is fulfilled. It is worth noting that in MLSD the merging condition is $[e(z)]_{l}^{l+\nu-1}=0$, which is exactly the condition (6) for the first-event error at the next step. Conversely, for $\mu<\nu$, when the binary test between $[\tilde{a}(z)]_{0}^{l-1}$ and $[\tilde{a}(z)+e(z)]_{0}^{l-1}$ takes place, there is at least one nonzero coefficient, namely $e_{l-1} \neq 0$, and at most $\nu-\mu$ nonzero coefficients in the polynomial $[e(z)]_{l+\mu-\nu}^{l+\mu-1}$. Hence, when the decision is $[\hat{a}(z)]_{0}^{l-1}=[\tilde{a}(z)+e(z)]_{0}^{l-1}$, the condition (6) for the first-event error is not satisfied at the next step after the binary test.

When MLSD is considered, the BER is upperbounded by attaching the Hamming weight of the error sequence to each term in the sum [9]:

$$
\mathrm{BER} \leq \sum_{e(z) \in \mathcal{E}} w_{e} 2^{-w_{e}} P(e)
$$

Due to error propagation, (10) does not hold true when $\mu<\nu$. To overcome this difficulty, an ideal DDFSD is considered in $[2,8]$, where error propagation is neglected. More precisely, when the DDFSD decides $[\hat{a}(z)]_{0}^{l-1}=[\tilde{a}(z)+e(z)]_{0}^{l-1}$, some demon cancels the errors $[e(z)]_{l-\nu+\mu}^{l-1}$ from the memory of the DFE associated to $[\hat{a}(z)]_{0}^{l-1}$. For this ideal DDFSD the error burst terminates when the wrong decision is taken, and $w_{e}$ is exactly the number of errors contained in the burst, hence the upper bound (10) holds true. When the effect of the errors $[e(z)]_{l-\nu+\mu}^{l-1}$ on the $\nu-\mu$ decisions at time $l+\mu, \cdots, l+\nu-1$ is negligible, it is proposed in [8] to approximate the BER of the true DDFSD as the RHS of (10).

\section{A. Computation of the Pairwise Error Probability}

The pairwise error probability is the probability of error in the binary test

$$
\sum_{k=0}^{l+\mu-1}\left(x_{k}-\sum_{j=0}^{\nu} d_{j} \tilde{a}_{k-j}\right)^{2} \gtrless \sum_{k=0}^{l+\mu-1}\left(x_{k}-\sum_{j=0}^{\nu} d_{j}\left(\tilde{a}_{k-j}+e_{k-j}\right)\right)^{2},
$$

where $[e(z)]_{0}^{l-1}$ belongs to the set $\mathcal{E}$ described in (8), and (6) and (9) are assumed. From the geometrical perspective, the decision boundary is the hyperplane between the two points that represent $[\tilde{a}(z)]_{0}^{l-1}$ and its competitor in the $(l+\mu)$ dimensional decision space. The direction that joins the two mentioned points is hereafter called output error, and is represented by the polynomial

$$
e_{o}(z)=[e(z) d(z)]_{0}^{l+\mu-1} .
$$

Note that, in contrast to MLSD, where $e_{o}(z)=$ $[e(z) d(z)]_{0}^{l+\nu-1}=e(z) d(z)$, in the DDFSD the decision is premature because the last $\nu-\mu$ samples of the output error are truncated. Using the $z$-transform, the binary test takes the form

$$
\left[u(z) u\left(z^{-1}\right)\right]_{0} \gtrless\left[\left(u(z)-e_{o}(z)\right)\left(u\left(z^{-1}\right)-e_{o}\left(z^{-1}\right)\right)\right]_{0},
$$

where $u(z)$ is the $z$-transform of the distortion sequence (5) in the decision space:

$$
u(z)=[x(z)-\tilde{a}(z) d(z)]_{0}^{l+\mu-1} .
$$


The binary test (12) is rewritten as

$$
\frac{\left[e_{o}\left(z^{-1}\right) u(z)\right]_{0}}{\delta_{e}} \gtrless \frac{\delta_{e}}{2},
$$

where

$$
\delta_{e}^{2}=\left[e_{o}(z) e_{o}\left(z^{-1}\right)\right]_{0},
$$

is the squared Euclidean distance between the competitors. Geometrically, the LHS of (14) is the projection of the distortion along the output error. The error occurs when such a projection, which is hereafter called

$$
\phi_{e}=\frac{\left[e_{o}\left(z^{-1}\right) u(z)\right]_{0}}{\delta_{e}},
$$

exceeds half the Euclidean distance between the competitors. The pairwise error probability is

$$
P(e)=\int_{\delta_{e} / 2}^{\infty} f_{\phi_{e}}(x) d x
$$

where $f_{\phi_{e}}(x)$ is the probability density function of $\phi_{e}$. The calculation of $f_{\phi_{e}}(x)$ proceeds by considering the distortion as the sum of ISI and noise. Specifically, the projection of the noise along the output error is

$$
\zeta_{e}=\frac{\left[e_{o}\left(z^{-1}\right) n(z) p(z)\right]_{0}}{\delta_{e}}
$$

where $n(z)$ is zero mean Gaussian noise with autocorrelation $\sigma^{2} r(z)$. The probability density function $f_{\zeta_{e}}(x)$ is Normal, with mean

$$
m_{\zeta_{e}}=0,
$$

and variance

$$
\sigma_{\zeta_{e}}^{2}=\frac{\sigma^{2}}{\delta_{e}^{2}}\left[e_{o}\left(z^{-1}\right) p(z) r(z) p\left(z^{-1}\right) e_{o}(z)\right]_{0} .
$$

The projection of the ISI along the output error is

$$
\begin{aligned}
\psi_{e} & =\frac{1}{\delta_{e}}\left[e_{o}\left(z^{-1}\right)(\tilde{a}(z)(r(z) p(z)-d(z)))\right]_{0} \\
& =-\frac{\sigma^{2}}{\delta_{e}}\left[e_{o}\left(z^{-1}\right) p(z) \tilde{a}(z)\right]_{0}=[c(z) \tilde{a}(z)]_{0}
\end{aligned}
$$

where $c(z)$ is the polynomial of the coefficients of the ISI. The probability density function $f_{\psi_{e}}(x)$ can be computed from the coefficients of the ISI. In the section devoted to the experimental results, we adopt the method proposed in [10]. Due to space limitation, the derivation of the mean value and of the variance of $\psi_{e}$ are omitted. The results are ${ }^{1}$

$$
m_{\psi_{e}}=\frac{2 \sigma^{2} w_{e}}{\delta_{e}}
$$

\footnotetext{
${ }^{1}$ Looking at (22), one might suspect that the term $4 \sigma^{4} w_{e} / \delta_{e}^{2}$ should be emended as $4 \sigma^{4} w_{e}^{2} / \delta_{e}^{2}$. This footnote is to confirm to the referees that (22) reports the exact result.
}

$$
\sigma_{\psi_{e}}^{2}=\left[c(z) c\left(z^{-1}\right)\right]_{0}-\frac{4 \sigma^{4} w_{e}}{\delta_{e}^{2}}
$$

Since ISI and noise are independent random variables, the probability density function of $\phi_{e}$ is

$$
f_{\phi_{e}}(x)=f_{\psi_{e}}(x) \otimes f_{\zeta_{e}}(x),
$$

where $\otimes$ denotes the convolution. Taking into account that the mean value of the Gaussian noise is zero, one has

$$
m_{\phi_{e}}=m_{\psi_{e}}=\frac{2 \sigma^{2} w_{e}}{\delta_{e}} .
$$

Using $p(z)\left(r(z)+\sigma^{2}\right) p\left(z^{-1}\right)=1$, for $\sigma_{\phi_{e}}^{2}$ one gets

$$
\sigma_{\phi_{e}}^{2}=\sigma_{\zeta_{e}}^{2}+\sigma_{\psi_{e}}^{2}=\sigma^{2}\left(1-\frac{4 \sigma^{2} w_{e}}{\delta_{e}^{2}}\right)
$$

\section{B. Truncation of the Union Bound}

In a brute force approach to performance evaluation, one should compute (23) and (16) for all the error polynomials up to a length such that convergence of the sum (7) is attained. A more sensible approach is to select those error polynomials that dominate the sum, and to compute

$$
\text { FEER } \approx \sum_{e(z) \in \mathcal{E}_{M}} 2^{-w_{e}} P(e)
$$

where $\mathcal{E}_{M}$ is the subset of $\mathcal{E}$ that contains the $M$ polynomials that dominate the sum. In principle, one should produce a list by ordering in descending order the terms that appear in the sum (7), and then should truncate the list to the first terms. However, at moderate-to-high SNR the coefficient $2^{-w_{e}}$ is dominated by $P(e)$, and ISI is dominated by the Gaussian noise [7], yielding the approximation

$$
P(e) \approx Q\left(\sqrt{\mathrm{SDR}_{e}}\right)
$$

where

$$
Q(x)=\frac{1}{\sqrt{2 \pi}} \int_{x}^{\infty} e^{-\frac{u^{2}}{2}} d u
$$

and $\mathrm{SDR}_{e}$ is the Signal-to-Distortion Ratio relevant to the specific $e(z)$. Using (24) and (25), for $\mathrm{SDR}_{e}$ one gets

$$
\operatorname{SDR}_{e}=\frac{\left(\delta_{e}-2 m_{\phi_{e}}\right)^{2}}{4 \sigma_{\phi_{e}}^{2}}=\frac{\delta_{e}^{2}-4 \sigma^{2} w_{e}}{4 \sigma^{2}} .
$$

Hence one can produce a large list of error polynomials ordered by increasing $\mathrm{SDR}_{e}$, and then compute $2^{-w_{e}} P(e)$ for each element of the list to produce a re-ordered list, which can be used in (26). Equation (28) makes it possible to produce the ordered list by exploiting the iteration

$$
i_{k}=i_{k-1}+\left(\sum_{j=0}^{\nu} d_{j} e_{k-j}\right)^{2}-\sigma^{2} e_{k}^{2}, k=0,1, \ldots, l+\mu-1,
$$




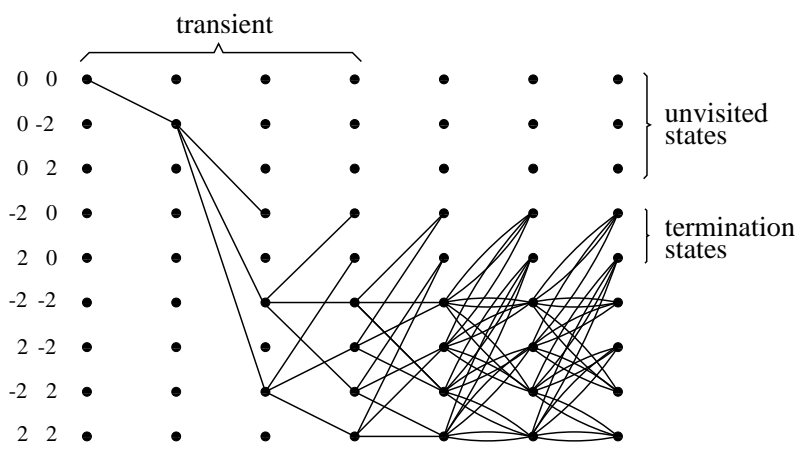

Fig. 2. Error trellis for $\nu=2, \mu=1$, two survivors per state.

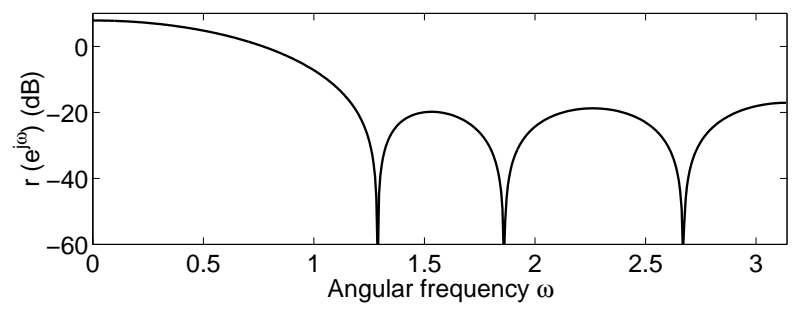

Fig. 3. Spectrum of the channel: $[r(z)]_{0}^{\nu}=0.9978+0.9185 z^{-1}+$ $0.7304 z^{-2}+0.4881 z^{-3}+0.2674 z^{-4}+0.1112 z^{-5}+0.031 z^{-6}$.

$i_{-1}=0, i_{l+\mu-1}=\delta_{e}^{2}-4 \sigma^{2} w_{e}, e_{-\nu}=\cdots=e_{-1}=e_{l}=$ $\cdots=e_{l+\mu-1}=0$. Several methods based on the iterative computation of the metric have been proposed in the past to produce the ordered list for MLSD. Discussing these methods is out of the scope of this paper. We pick the baseline Viterbi algorithm with $3^{\nu}$ states described in [11], and use in a straightforward manner the iteration (29) in the branch metric. In the formulation of [11], the algorithm finds the error polynomial at minimum SDR in MLSD, and can be applied only to noncatastrophic channels. To determine the first $2 M$ error polynomials, we take inspiration from the generalized Viterbi algorithm of [12]. Note that, since $e(z)$ and $-e(z)$ give the same $\mathrm{SDR}_{e}$, allowing $M$ survivors per state in the algorithm of [12] is sufficient to find the first $2 M$ polynomials. Recalling (9), when the DDFSD is considered one has to handle the states of the type $\left(e_{l-\nu+\mu}, \ldots, e_{l-1}, \mu-z\right.$ eros $), e_{l-1} \neq 0$, as termination states. For, it is sufficient to cancel from the complete trellis all the transitions that diverge from all the termination states. Of course, all the states that are reached only from termination states will remain unvisited after an initial transient of $\nu+1$ steps, and, after the transient, these states can be removed from the trellis. A pictorial description of this trellis is reported in Fig. 2.

\section{EXPERIMENTAL RESULTS}

To substantiate the results obtained in the previous section, we adopt as a benchmark the time discrete white Gaussian channel with $\nu=6$ studied in [13]. The spectrum $r\left(e^{j \omega}\right)$ is depicted in Fig. 3 versus angular frequency $\omega$. The feature of this channel is that no channel having lower minimum distance with the same $\nu$ exists. Fig. 4 reports the convergence of the union bound on the FEER for the MMSE-DDFSD with

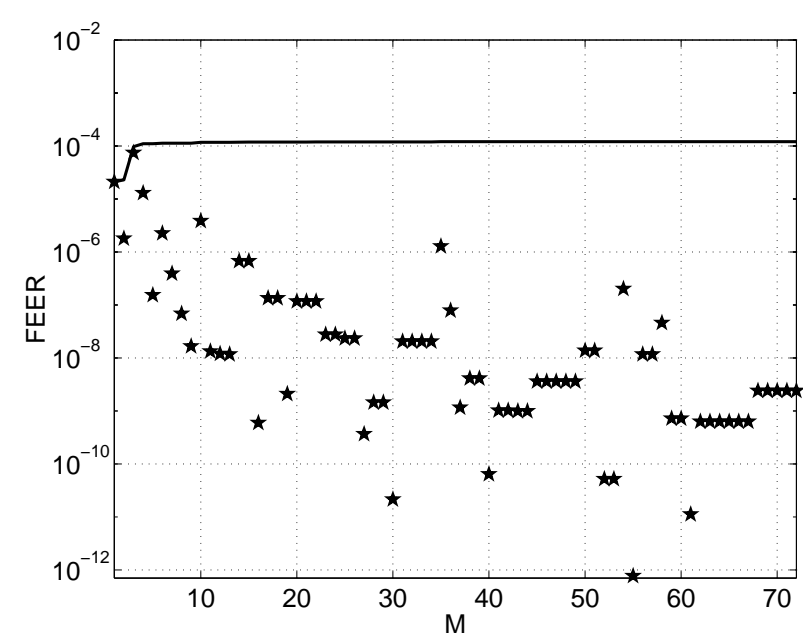

Fig. 4. The straight line is the union bound truncated to the first $2 M$ error polynomials versus $M$, ordered by SDR. The computer simulation gives FEER $=1.0710^{-4}$. The star is the contribution of the $M$-th pair of polynomials $e(z),-e(z)$, to the sum. The Viterbi algorithm terminates at the seventyfirst step.

$\mu=4$ at $\mathrm{SNR}=r_{0} / \sigma^{2}=20 d B$. The 144 error polynomials at lower SDR have been found by the algorithm described in the previous section, and the contribution $2^{-w_{e}} P(e)$ of each pair $e(z),-e(z)$ in the sum is reported in the figure. The 18 error polynomials at lower SDR are listed in Table I, where $P(e)$ is computed as in [10]. Fig. 5 reports the FEER versus SNR for MLSD and for the MMSE-DDFSD and the W-DDFSD with $\mu=0$ and $\mu=4$. In the simulations, the FEER is measured by collecting at least 100 events. In the computation of the truncated upper bound, the list ordered by SDR is reordered by $2^{-w_{e}} P(e)$, and the first 18 terms are used. From Fig. 5, one realizes that truncation to the first 18 terms virtually gives the upper bound. Fig. 6 reports the BER versus SNR with the same parameters as in Fig. 5. The straight line is the RHS of (10) truncated to the first 18 terms after re-ordering by $w_{e} 2^{-w_{e}} P(e)$. The figure shows that the approximation is more accurate for the DDFSD than for the DFE, while for MLSD the first 18 terms virtually give the upper bound. This is an expected result, since the impact of error propagation on the BER diminishes passing from the pure DFE to MLSD.

\section{CONCLUSIONS}

The main result of the paper is the performance evaluation of the MMSE-DDFSD. Equation (28) makes it feasible to produce a large list ordered by increasing SDR of the error polynomials that dominate the FEER of the MMSE-DDFSD. The FEER is then evaluated by truncating the union bound, where truncation is such that only the terms that dominate the sum are taken into account. Evaluation of the BER is complicated by the error propagation induced by the per-survivor DFE. However, an approximation to the BER can be obtained if error propagation is neglected. Such an approximation fairly fits the simulation results only for moderate reduction of complexity. 
TABLE I

FIRST 18 ERROR POLYNOMIALS FOR THE MMSE-DDFSD AND FOR THE W-DDFSD WITH $\mu=4$ AT SNR $=20 d B$. ONLY THE 9 POLYNOMIALS BEGINNING WITH $e_{0}=-2$ ARE LISTED. IN THIS SPECIFIC EXAMPLE, IT HAPPENS THAT THE FIRST 18 POLYNOMIALS ARE THE SAME FOR THE W-DDFSD AND FOR THE MMSE-DDFSD.

\begin{tabular}{|c|c||c||l|}
\hline$P_{M M S E}(e)$ & $\mathrm{SDR}_{e, M M S E}[\mathrm{~dB}]$ & $\mathrm{SDR}_{e, W}[\mathrm{~dB}]$ & Coefficients of $e(z)$ \\
\hline \hline $6.77 \cdot 10^{-4}$ & 10.11 & 9.04 & $-222-2-22$ \\
$2.30 \cdot 10^{-4}$ & 10.89 & 10.00 & $-222-2-222-2$ \\
$1.49 \cdot 10^{-4}$ & 11.16 & 10.35 & -22 \\
$1.04 \cdot 10^{-4}$ & 11.38 & 10.61 & $-22000-22$ \\
$0.79 \cdot 10^{-4}$ & 11.54 & 10.80 & $-222-2-222-2-22$ \\
$0.72 \cdot 10^{-4}$ & 11.59 & 10.86 & $-22000-22000-22$ \\
$0.50 \cdot 10^{-4}$ & 11.79 & 11.10 & $-22000-22000-22000-22$ \\
$0.35 \cdot 10^{-4}$ & 11.98 & 11.32 & $-22000-22000-22000-22000-22$ \\
$0.34 \cdot 10^{-4}$ & 12.00 & 11.34 & $-222-2-2202-2-222-2$ \\
\hline
\end{tabular}

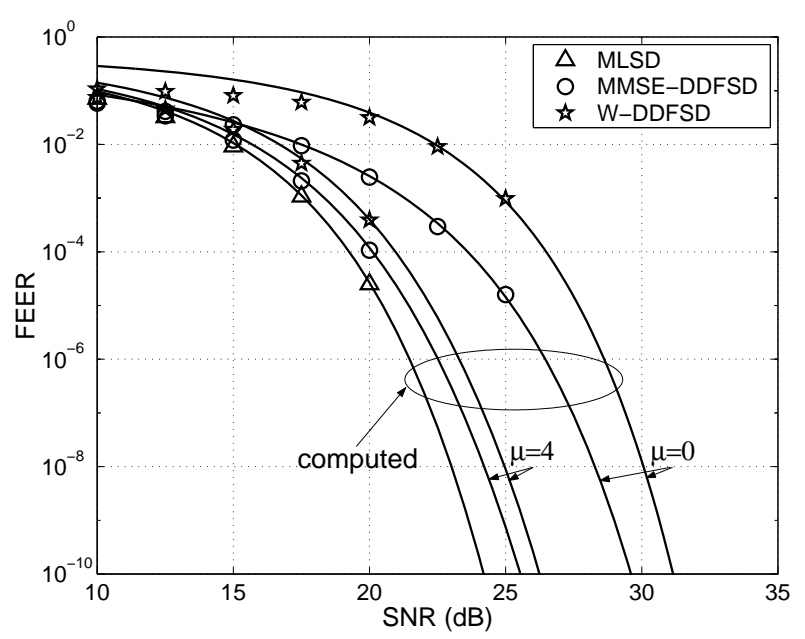

Fig. 5. FEER versus SNR. The straight line is the union bound truncated to the first 18 terms.

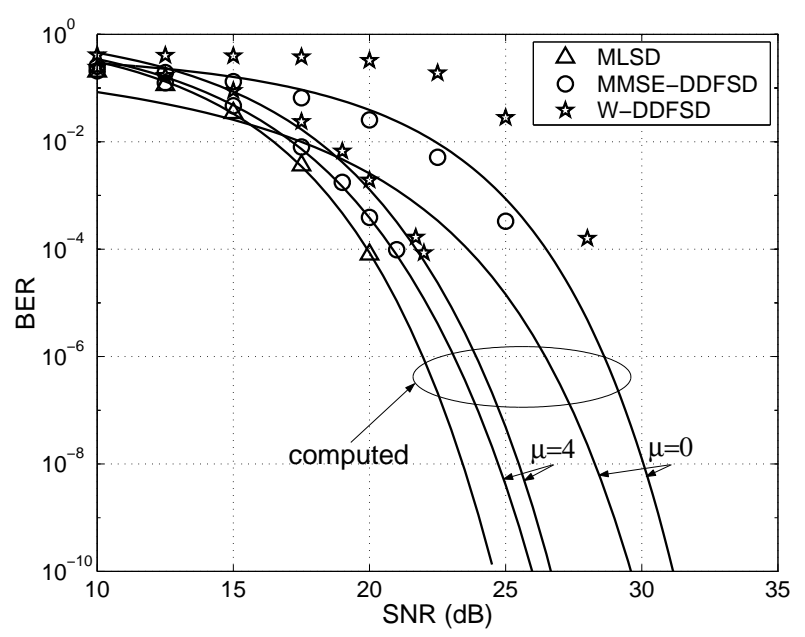

Fig. 6. BER versus SNR. The straight line is the approximation truncated to the first 18 terms.

\section{REFERENCES}

[1] R. Raheli, A. Polydoros, and C.-K. Tzou, "Per-survivor processing: a general approach to MLSE in uncertain environments," IEEE Trans. Commun., vol. 43, pp. 354-364, Feb./Mar./Apr. 1995.

[2] A. Duel-Hallen and C. Heegard, "Delayed decision-feedback sequence estimation," IEEE Trans. Commun., vol. 37, pp. 428436, May 1989.

[3] W. H. Gerstacker, and J. B. Huber, "Improved equalization for GSM mobile communications," Proc. Int. Conf. Telecommun., pp. 128-131, Istanbul, Apr. 1996.

[4] M. Schmidt, and G. P. Fettweis, "Fractionally-spaced prefiltering for reduced state equalization," Proceedings of Globecom '99, vol. 5, pp. 2291-2295, Rio de Janeiro, Brazil, Dec. 1999.

[5] M. Magarini, A. Spalvieri, and G. Tartara, "Comparison between two methods for delayed decision feedback sequence estimation," Proceedings of PIMRC 2000, vol.2, pp. 1300-1304, London, U.K., Sept. 2000.

[6] J. Salz, "Optimum mean-square decision feedback equalization," Bell Syst. Tech. J., vol. 52, pp. 1341-1373, Dec. 1973.

[7] J. M. Cioffi, G. P. Dudevoir, M. V. Eyuboglu, and G. D. Forney Jr., "MMSE decision-feedback equalizers and coding-part I: equalization results, "IEEE Trans. Commun., vol. 43, pp. 2582-2594, Oct. 1995.

[8] W. Sheen and G. D. Stüber, "Error probability for reduced-state sequence estimation," IEEE J. Select. Areas Commun., vol. 10, pp. 571-578, April 1992.

[9] A. J. Viterbi and J. K. Omura, " Principles of Digital Communication and Coding," McGraw-Hill, pp. 278-279, 1979.

[10] J. C. Vanelli and N. M. Shehadeh, "Computation of bit error probability using the trapezoidal integration rule," IEEE Trans. Commun., vol. 22, pp. 331-334, March 1974.

[11] E. A. Lee and G. D. Messerschmitt, "Digital Communication," Kluwer, pp. 277-278, 1993.

[12] T. Hashimoto, "A list-type reduced-constraint generalization of the Viterbi algorithm," IEEE Trans. Inform. Theory, vol. 33, pp. 866-876, Nov. 1987.

[13] R. R. Anderson and G. J. Foschini, "The minimum distance for MLSE digital data systems of limited complexity," IEEE Trans. Inform. Theory, vol. 21, pp. 544-551, Sept. 1975. 